\title{
EDITORIAL
}

\section{Investigación en tiempos de pandemia}

Los tiempos grises que vivimos en la actualidad golpeó a la humanidad haciéndola vulnerable, cuando pensamos que teníamos todo controlado o casi controlado, un organismo microscópico le ha negado esa posibilidad a humanidad. La magnitud de la pandemia ha puesto en jaque a la ciencia poniendo el acelerador a los investigadores que tienen la urgente tarea de encontrar el antiviral que signifique volver a la nueva normalidad.

El reto de derrotar a la pandemia trae consigo ingentes esfuerzos de la comunidad científica, no obstante para tener éxito en estas intenciones es necesario contar con recursos y logística que en nuestros países son problemas que arrastramos desde siempre, en ese escenario existen esfuerzos gubernamentales y de empresas privadas por financiar investigaciones orientadas principalmente a encontrar el antiviral contra el SRAS-CoV-2 más conocido como COVID - 19 o coronavirus, en esa dirección las bases de datos especializadas han realizado una apertura a sus contenidos permitiendo acceso abierto, democratizándose así el conocimiento, los académicos reconocen este escenario como una nueva oportunidad para relanzar sus actividades con el compromiso que la profesión les asigna, asumiendo nuevas estrategias que puedan dar respuestas más eficaces a problemas que la sociedad plantea con la finalidad que su labor sea significativo

Frente a las actuales circunstancias de trabajo desde el hogar, las estrategias de investigación deben descansar en un importante soporte tecnológico orientado a plataformas virtuales y aunque las limitaciones pueden ser incalculables, es evidente que para sobrevivir; académica y científicamente el investigador debe avanzar paralelamente con el conocimiento tecnológico.

Es imposible pensar que después de este hito, las actividades de investigación no se desarrollen usando plataformas activas que permiten realizar trabajos colaborativos entre equipos multidisciplinarios que seguramente enriquecerán mejor las investigaciones. Por tanto otra forma de democratizar la información es compartir información a tiempo real con pares de cualquier parte del mundo.

Si bien es cierto que en la actualidad los científicos están abocados en derrotar al virus, los que realizan investigaciones en otras disciplinas buscan entender las consecuencias de la pandemia en otros contextos, no menos importante son los problemas que se atravesaba y que son necesarios de atender tales como el cambio climático, igualdad de género, eficiencias en la producción, acceso a recurso, etc., que no se han ido. Por tanto es imperativo que se siga abordando ese tipo de problemáticas, esa es la tarea irrenunciable de este espacio académico.

Dr. Arcadio Atencio Vargas

Director revista Ingeniería Investiga

Decano Facultad Ingeniería 


\section{INGENIERÍA INUESTIGA}

\section{Director}

\section{Dr. Arcadio Atencio Vargas}

Decano Facultad de Ingeniería, Universidad Privada de Tacna

\section{EDITOR}

\section{Dr. Raul Cartagena Cutipa}

Coordinador Unidad de Investigación Facultad de Ingeniería, Universidad Privada de Tacna

\section{Equipo Editorial}

\section{Dr. Noribal Zegarra Alvarado}

Escuela de Ingeniería Civil, Universidad Privada de Tacna

\section{Sc. Luis Alfredo Fernández Vizcarra}

Escuela de Ingeniería de Sistemas, Universidad Privada de Tacna

\section{Mtro. Milagros Herrera Rejas}

Escuela de Ingeniería Ambiental, Universidad Privada de Tacna

\section{Sc. Julio Málaga Núñez}

Ingeniería de Electrónica, Universidad Privada de Tacna

\section{MBA. Luis Espinoza Villalobos}

Escuela de Ingeniería Industrial, Universidad Privada de Tacna

\section{Dr. Samuel Banda Arrieta}

Universidad Autónoma de Chapingo - México

\section{Dr. Alberto Vega Muñoz}

Escuela de Administración y Gestión, Universidad Tecnológica de Chile

\section{PhD. Juan Marco Aro Aro}

Universidad Nacional del Altiplano, Perú

\section{Dr. Américo Ariel Rubín de Celis Vidal \\ Universidad Nacional de Moquegua, Perú}

\section{Dr. Jaime del Carpio Salinas}

Asociación Civil Universidad de Ciencias y Humanidades UCH, Perú

\section{Dr. Gilmar Lizana Puelles}

Ingeniería Industrial, Universidad Ricardo Palma, Perú.

\section{Consejo Consultivo}

\section{Dr. Javier Masías Astengo}

Director de Oficina de Planificación, Presupuesto y Desarrollo, MINCETUR, Perú

\section{Dr. Juan Ríos Segura}

Jefe de Construcción, Universidad Nacional de Ingeniería, Perú.

\section{Dr. Ángel Canales Gutiérrez}

Facultad de Ciencias Biológicas, Universidad Nacional del Altiplano, Perú.

Mg. José Alberto Acero Martínez

Universidad Nacional de Ingeniería, Perú.

\section{Mg. Wilson Edgar Silva Berríos}

Pontificia Universidad Católica el Perú (PUCP), Perú. Académico Principal del Departamento de Ingeniería del Área de estructuras de la PUCP.

\section{Mg. Wilson Silva Berrios}

Departamento de Ingeniería Área de Estudios, Pontificia Universidad Católica del Perú,

\section{Mg. José Martin Velásquez Vargas}

Pontificia Universidad Católica del Perú

\section{Dra. Sylvia Carolina Alcarzar Alay}

Vicerrectorado de Investigación, Universidad Nacional Jorge Basadre Grohmann, Tacna Perú.

\section{Mag. José Salgado Canal}

Coordinador de Investigación de Pregrado, Facultad de Ingeniería Civil, Universidad Nacional de Ingeniería, Perú.

\section{Mgr. Wilson Edgar Silva Berríos}

Pontificia Universidad Católica del Perú (PUCP), Perú. Académico Principal del Departamento de Ingeniería del Área de Estructuras de la PUC 


\section{PRESENTACIÓN}

"INGENIERÍA INVESTIGA", es la revista oficial para publicaciones científicas de la Facultad de Ingeniería de la Universidad Privada de Tacna. Su publicación se realiza en forma semestral, y son arbitradas por un equipo consultivo. Es de distribución gratuita. En "INGENIERÍA INVESTIGA", se publican artículos que corresponden a trabajos de investigación que se relacione con todas las áreas profesionales de ingeniería, (Ingeniería de sistemas, electrónica, civil, agroindustrial, ambiental, industrial y otros afines) y; que aporten al conocimiento de las ciencias. Los artículos para ser aceptados y publicados deberán ser originales, no debe haber sido publicado parcial o en su forma completa, ni presentados para su publicación en otra revista en forma impresa o electrónica. Los autores se responsabilizan del material presentado.

\section{EDITOR ADMINISTRATIVO Y RESPONSABLE DE INFORMACIÓN}

Dr. Raul Cartagena Cutipa. Coordinador Unidad de Investigación Facultad de Ingeniería, Universidad Privada de Tacna. Celular: 051- 952341032 Email: ingenieria.investiga@upt.edu.pe código postal: 126 Domicilio

Campus Capanique S/N

\section{DATOS DE LA REVISTA:}

URL : http://revistas.upt.edu.pe/ojs/index.php/ingenieria

ISSN Edición Online: 2708-3039

Servicio de información (índices y resúmenes) ingenieria.investiga@upt.edu.pe

Publicación semestral

Revista de Investigación de la Facultad de Ingeniería de la Universidad Privada de

Tacna

Se prohíbe la reproducción o transcripción total o parcial de los artículos contenidos en esta edición, sin autorización escrita de los autores. 


\section{INGENIERÍA INUESTIGA}

VOL. 2 NO 1 ENERO - JUNIO 2020

\section{CONTENIDO}

\section{Artículos originales}

FRECUENCIAS Y PERIODOS PREDOMINANTES DE SISMOS REGISTRADOS EN LA CIUDAD DE TACNA, MEDIANTE ANÁLISIS Y ESPECTRO DE FOURIER

FREQUENCIES AND PREDOMINANT PERIODS OF EARTHQUAKES REGISTERED IN THE CITY OF TACNA, BY MEANS OF ANALYSIS AND FOURIER SPECTRUM

Wilber Mendoza Ramírez, Dina Marlene Cotrado Flores, Joel Ticahuanca Mamani, Arleth Mendoza Chura

$160-175$

CARACTERIZACIÓN DE RESIDUOS SÓLIDOS EN EL CAMPUS CAPANIQUE DE LA UNIVERSIDAD PRIVADA DE TACNA

CHARACTERIZATION OF SOLID WASTE IN THE CAPANIQUE CAMPUS OF THE PRIVATE UNIVERSITY OF TACNA Richard Sabino Lazo Ramos y Milagros Herrera Rejas

$176-185$

DESARROLLO E IMPLEMENTACION DE UN SISTEMA INMÓTICO (IOT) DE GESTIÓN ENERGÉTICA PARA LA UNIVERSIDAD PRIVADA DE TACNA

DEVELOPMENT AND IMPLEMENTATION OF AN ENERGY MANAGEMENT IMMOTE SYSTEM (IOT) FOR THE PRIVATE UNIVERSITY OF TACNA

Christian Acero Catacora, Tito Ale-Nieto, Enrique Lanchipa Valencia y Jorge Deza

$186-198$

PROPUESTA DE FÓRMULA PARA LA DETERMINACIÓN DE LA DENSIDAD DE MUROS CONSTRUIDOS CON BLOCKER II

PROPOSAL FORMULA FOR THE DETERMINATION OF THE DENSITY OF WALLS BUILT WITH BLOCKER II

Dina Cotrado Flores

$199-210$

ESPECTROS DETERMINÍSTICOS Y PROBABILÍSTICOS PARA LA EVALUACIÓN DEL PELIGRO SÍSMICO EN ESTRUCTURAS DE LA REGIÓN DE TACNA

DETERMINISTIC AND PROBABILISTIC SPECTRUMS FOR THE EVALUATION OF SEISMIC HAZARD IN STRUCTURES OF THE TACNA REGION

Ever Rudy Ancco Huanacuni y Edgar Hipolito Chaparro Quispe

$211-224$

INTRODUCCIÓN DEL SISTEMA DE ADVERTENCIAS AL CONSUMIDOR BASADO EN OCTÓGONOS Y EL CONSUMO DE ALIMENTOS PROCESADOS EXCESIVOS EN NUTRIENTES

INTRODUCTION OF THE OCTOGON-BASED CONSUMER WARNING SYSTEM AND CONSUMPTION OF EXCESSIVE PROCESSED FOOD IN NUTRIENTS

Muriel Alférez Murias, Sebastián Alférez Murias y Raul Cartagena Cutipa

$225-245$ 
EVALUACIÓN DE MURO VERDE EN LA DISMINUCIÓN DE RUIDO Y SU PECEPCIÓN AMBIENTAL EN UN CENTRO EDUCATIVO

EVALUATION OF GREEN WALL IN THE DECREASE OF NOISE AND ITS ENVIRONMENTAL PERCEPTION IN AN EDUCATIONAL CENTER

Ana Claudia Zeballos Pimentel y Carmen Rosa Román Arce

$246-253$

CONTAMINACIÓN ACÚSTICA Y SU PERCEPCIÓN AMBIENTAL EN LA COMUNIDAD EDUCATIVA DEL CERCADO DE TACNA, 2019

NOISE POLLUTION AND ITS ENVIRONMENTAL PERCEPTION IN THE EDUCATIONAL COMMUNITY OF CERCADO DE TACNA, 2019

Antuanne Mariela Mamani Valdez y Marisol Mendoza Aquino

$254-264$

DISEÑO DE UNA RED FTTH PARA EL DISTRITO GREGORIO ALBARRACÍN LANCHIPA, TACNA

DESIGN OF FTTH NETWORK TO GREGORIO ALBARRACIN LANCHIPA DISTRICT, TACNA

Ernesto Antonio Rios De La Cruz, Huido Quino Huanacuni y Hugo Javier Rivera Herrera

$265-275$

CONDICIONES METEOROLÓGICAS Y MATERIAL PARTICULADO PM10 Y PM2.5 EN LA CONSTRUCCIÓN DEL HOSPITAL HIPÓLITO UNÁNUE, TACNA 2019

METEOROLOGICAL CONDITIONS AND PARTICULATE MATERIAL PM10 AND PM2.5 IN THE CONSTRUCTION OF THE HOSPITAL HIPÓLITO UNÁNUE, TACNA 2019

Diego Motocanche Ayala y Richard Sabino Lazo Ramos

$276-285$

EVALUACIÓN DE ALGUNOS PARÁMETROS FíSICOS DE Persea americana MEDIANTE UN EQUIPO DE MEDICIÓN DE POSTCOSECHA

EVALUATION OF SOME PHYSICAL PARAMETERS OF PERSEA AMERICANA IN POST-HARVEST MEASUREMENT EQUIPMENT

Raul Cartagena Cutipa, Percy Fermín Velázquez Ccosi, Danny Daniel Carhuaz Valdez y Hugo Javier Rivera Herrera

$286-293$

\section{Artículo de revisión}

TECNOLOGÍAS ECOEFICIENTES PARA LA VALORACIÓN DE RESIDUOS

AGROINDUSTRIALES EN FRUTAS Y HORTALIZAS

ECO-EFFICIENT TECHNOLOGIES FOR THE VALUATION OF AGRO-INDUSTRIAL WASTE IN FRUITS AND VEGETABLES

Marianne Hilda Cornejo Figueroa, Raul Cartagena Cutipa y Sylvia Carolina Alay Alcázar

$294-311$ 